\title{
Polycystic kidney disease concurrent with feline parvovirus and bacterial infections in domestic shorthair cat: a case report
}

\author{
Pauline Poh Ling Sim Lam ${ }^{1} \cdot$ Mohd Farhan Hanif Reduan ${ }^{1}$ (D) $\cdot$ Sabri Jasni ${ }^{1} \cdot$ Rumaizi Shaari $^{2} \cdot$ \\ Nurshahirah Shaharulnizim ${ }^{2}$ - Muhammad Luqman Nordin ${ }^{2}$ • Aziz Abd Rahman ${ }^{1}$ - Nadiah Syuhada Roslan ${ }^{2}$
}

Received: 19 April 2020 / Accepted: 22 September 2020 / Published online: 28 September 2020

(C) Springer-Verlag London Ltd., part of Springer Nature 2020

\begin{abstract}
Feline polycystic kidney disease (PKD) is an inherited disorder caused by the mutation of PKD1 gene that eventually lead to the development of chronic kidney disease. The latter condition causes hypertension and eventually progress into congestive heart failure. Feline parvovirus (FPV) is a highly contagious and often fatal disease infecting cats and other members of Felidae. An 8-month-old female domestic shorthair cat was presented with complaint of wound dehiscence a day after ovarian hysterectomy procedure. The wound at the suture site appeared necrotic, purulent with foul smell. The cat was found to have diarrhoea during the fixation of suture breakdown and, later, was tested positive with parvovirus infection. Complete blood count revealed anaemia, neutrophilia, lymphopenia and thrombocytosis. Biochemistry profiles showed hypoproteinaemia and elevated of urea and creatinine. The cat was hospitalised, and symptomatic treatments were given. During hospitalisation, the cat showed symptoms of polydipsia and polyuria and found dead 2 days later. Post-mortem findings demonstrated the cat had oral ulceration, thoracic effusion, fibrinopleuropneumonia, pericardial effusion, left ventricular hypertrophy and right ventricular dilation, chronic passive liver congestion, mesenteric lymphadenomegaly, intestinal haemorrhage, adrenomegaly and polycystic kidney. Histopathological evaluation revealed fibrinous pleuropneumonia, pulmonary atelectasis, emphysema and oedema, hypertrophic cardiomyopathy, hepatic necrosis, splenic necrosis, intestinal necrosis, renal necrosis and renal polycystic. Staphylococcus aureus and Escherichia coli were isolated from bronchus swab and intestinal segment, respectively. Polymerase chain reaction (PCR) revealed parvovirus infection. The cat was definitely diagnosed with polycystic kidney disease concurrent with parvoviral and secondary bacterial infections.
\end{abstract}

Keywords Chronic kidney disease $\cdot$ Congestive heart failure $\cdot$ Feline parvovirus $\cdot$ Polycystic kidney disease

\section{Introduction}

Polycystic kidney disease (PKD) is an inherited genetic disease in cats and has a high prevalence in Persian cats and Persian-related cats and infrequent in mixed breeds (Volta et al. 2010). Purebred cat has a prevalence of $20 \%$ occurrence while Persian-type breed constitutes of the remaining $80 \%$, making PKD a commonly found inherited disease for cats

Mohd Farhan Hanif Reduan

farhan.h@umk.edu.my

1 Department of Para Clinical Studies, Faculty of Veterinary Medicine, Universiti Malaysia Kelantan, Pengkalan Chepa, 16100 Kota Bharu, Kelantan, Malaysia

2 Department of Clinical Studies, Faculty of Veterinary Medicine, Universiti Malaysia Kelantan, Pengkalan Chepa, 16100 Kota Bharu, Kelantan, Malaysia
(Lyons et al. 2004). It is characterised by the presence of more than one fluid-filled cysts in the kidneys. The renal cysts tend to multiply in numbers and grow in size over time as the animal ages. This leads to progressing deterioration of the kidney tissue due to pressure necrosis, causing chronic kidney failure and, eventually, the death of the cat (Wills et al. 2009).

The progressive development of the polycystic kidney disease (PKD) depends on individual cats; kittens with severe cyst involvement may die at the age of 8 weeks due to kidney failure. However, the disease is usually subclinical until they are middle age or older (Barrs et al. 2001). Diagnosis of PKD is mainly by ultrasonography showing multiple anechoic structures within the renal parenchyma (Phoon et al. 2015). Polycystic kidney disease is one of the causes for the development of chronic kidney disease (CKD) in cats which cause deterioration of glomerular filtration rate and metabolic waste excretion.

Feline parvovirus (FPV) is a highly contagious and often fatal disease infecting cats and other members of Felidae. It is 
highly suspected if an unvaccinated cat exhibits clinical signs of watery to haemorrhagic diarrhoea, fever, depression and anorexia. Infected cats mostly die of secondary bacterial infection, sepsis, dehydration and disseminated intravascular coagulopathy (Hartmann 2017). Infected cats with severe leukopenia, thrombocytopenia, hypoalbuminemia and hypokalaemia will have a poor prognosis of FPV infection (Barrs 2019). This case report aims to highlight the diagnosis of polycystic kidney disease concurrent with parvoviral and bacterial infections in domestic shorthair cat.

\section{Case history}

An 8-month-old female domestic shorthair stray cat was brought to a private veterinary clinic for ovariohysterectomy procedure. Vaccination and deworming status were not up to date. However, the suture broke down on day 1 of post-op. On day 6 of post-op, the cat was presented to the University Malaysia Kelantan Veterinary Clinic with the complaint of a foul-smelling wound on the incision site. Physical examination revealed the presence of purulent necrotic tissue at the suture site. Surgical correction was carried out and Penrose drainage was placed. Upon the procedure, the cat showed episodes of watery diarrhoea and was diagnosed with parvovirus infection using SensPERT® parvovirus rapid test kit. The cat was hospitalised in the isolation unit where his condition deteriorates to severely dehydrated, lateral recumbency, tachypnoea, bradycardia, polydipsia, polyuria and stupor.

Complete blood count revealed the cat was having mild macrocytic normochromic anaemia (6.07, RR 6.5-10.0, 10\% $\mu \mathrm{l})$, neutrophilia $\left(16.7\right.$, RR $\left.2.0-8.0,10^{3} / \mu \mathrm{l}\right)$, lymphopenia (3.2, RR 5.7-8.9, $\left.10^{3} / \mu \mathrm{l}\right)$, monocytopenia $(0.3$, RR $2.2-4.0$, $\left.10^{3} / \mu \mathrm{l}\right)$ and thrombocytosis (1143, RR 300-800, 10 $\left.3 / \mu \mathrm{l}\right)$ (Abdallah et al. 2020a, b; Neamat-Allah et al. 2020). The serum biochemistry showed hypoproteinaemia (4.0, RR 5.7-8.9 g/dl) and elevated urea $(50, \mathrm{RR} 16-36, \mathrm{mg} / \mathrm{dl})$ and creatinine (5.3, RR 0.8-2.4, mg/dl) parameters (Abdallah et al. 2020a, b). The cat was treated with antibiotics; enrofloxacin $(5 \mathrm{mg} / \mathrm{kg})$ and metronidazole $(10 \mathrm{mg} / \mathrm{kg})$, nonsteroidal anti-inflammatory drugs; meloxicam $(0.3 \mathrm{mg} / \mathrm{kg})$, gastric protectant; ranitidine $(2.5 \mathrm{mg} / \mathrm{kg})$, gastrointestinal absorbent; kaolin-pectin, iron supplement; and vitamin B complex supplements. Normal saline was administered intravenously to rehydrate the patient. Besides all the medications given, the cat was found dead on day 5 of hospitalisation. Post-mortem was later conducted.

The post-mortem findings showed the cat had a poor body condition, oral ulceration, abdominal serous fat atrophy, thoracic effusion, fibrinopleuropneumonia, pulmonary oedema, tracheitis, pericardial effusion, left ventricular hypertrophy and right ventricular dilation, chronic passive liver congestion, splenic contraction, mesenteric lymphadenomegaly, intestinal haemorrhage, adrenomegaly and polycystic kidney (Figs. 1 and 2).

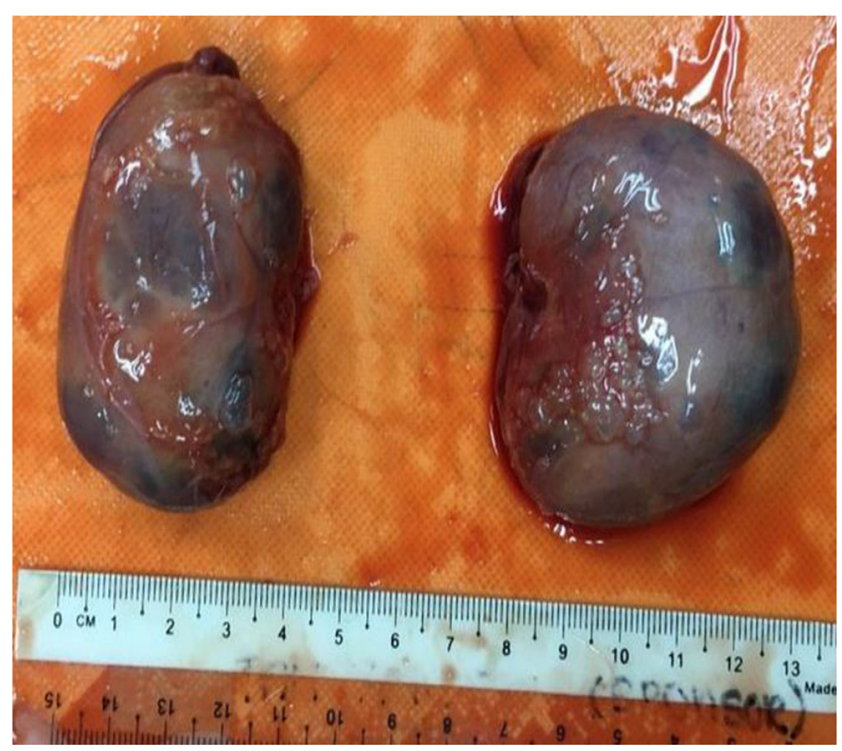

Fig. 1 The kidneys had multifocal dark red, fluid-filled cysts (arrows) that form upon palpation and are observable from the renal capsule. The left kidney was more distended and rounded at the renal capsule

Staphylococcus aureus and E. coli were isolated from bronchus swabs and intestine, respectively. Pleural fluid was found to be transudate through Rivalta's test. Molecular testing via polymerase chain reaction (PCR) technique sampled from a loop of intestine was found to be negative for coronavirus, but positive for parvovirus infection.

Haematoxylin and eosin (H\&E) staining on the lung tissue showed marked distension of the alveoli with rupture of some of the alveolar septa. Oedema fluid and fibrins were found to be present within the alveolar space. In the heart, there was presence of multiple box-car nuclei indicating enlarged cardiomyocytes with non-proteinaceous oedema of the cardiomyocytes. The liver showed diffuse hepatocytes with karyolytic necrosis, loss of liver sinusoidal architecture and central vein necrosis. In the spleen, there was diffuse splenic karyolytic necrosis. The intestinal tissue showed sloughed off and shortened intestinal villi and intestinal crypts epithelial cells and villi necrosis. The kidney tissue showed multiple cysts with homogenous fluid in the renal parenchyma and diffused loss of renal structural details due to the presence of space-occupying cysts (Fig. 3). The cysts were lined by a single layer of epithelial cells (Fig. 4). Some of the epithelial cells had sloughed off into the lumen while some had undergone karyolytic necrosis due to pressure necrosis (Fig. 5). The renal tubular epithelial cells showed pyknosis and karyolysis and some renal tubular cells showed hydropic degeneration, causing the swelling of the cells (Fig. 6).

\section{Discussion}

Polycystic kidney disease (PKD) is the most prominent congenital disease that is commonly found in Persian cats (38\%) 


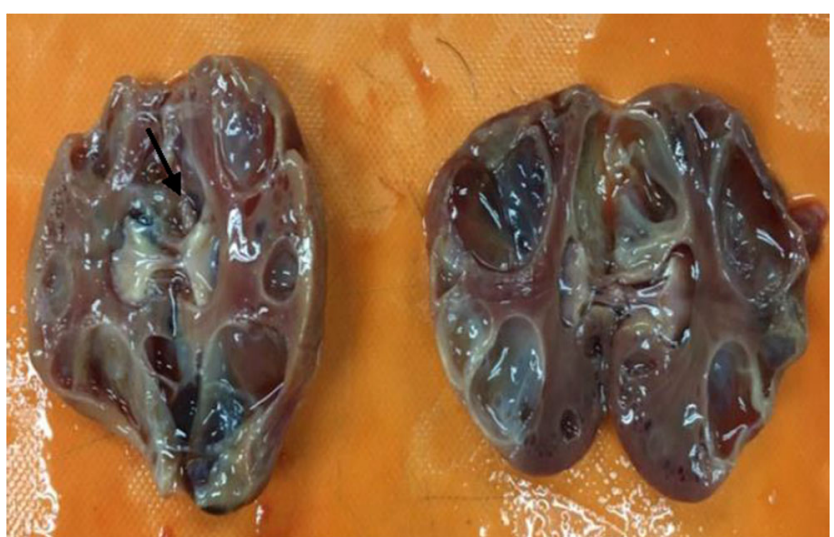

Fig. 2 Upon dissecting, yellowish fluid oozed out from the cyst and there were multiple irregular border cysts (arrows)

worldwide, Persian cross cats and very rarely in other commercial breeds (Lyons et al. 2004). Cats carry a mutation of PKD1 genes that had been identified as causative for PKD. It had been identified in heterozygous state in all 48 different breeds of cats including Persians, Siamese and domestic shorthairs. No homozygous cats had been identified from DNA testing, suggesting that the mutation is embryogenic lethal (Lyons et al. 2004).

Polycystic kidney disease produces histological changes such as an increase in the number and size of cyst in the renal parenchyma, necrosis and degeneration of renal tubular epithelial cells as observed in this case. Progression of these tissue injuries often results in CKD-associated complications such as hypertension, congestive heart failure and chronic anaemia (Brown et al. 2016). Hypertension caused by CKD can be due to salt retention due to decrease glomerular filtration rate (GFR), the inappropriate activity of renin-angiotensin system (RAS) activation and sympathetic nervous system as

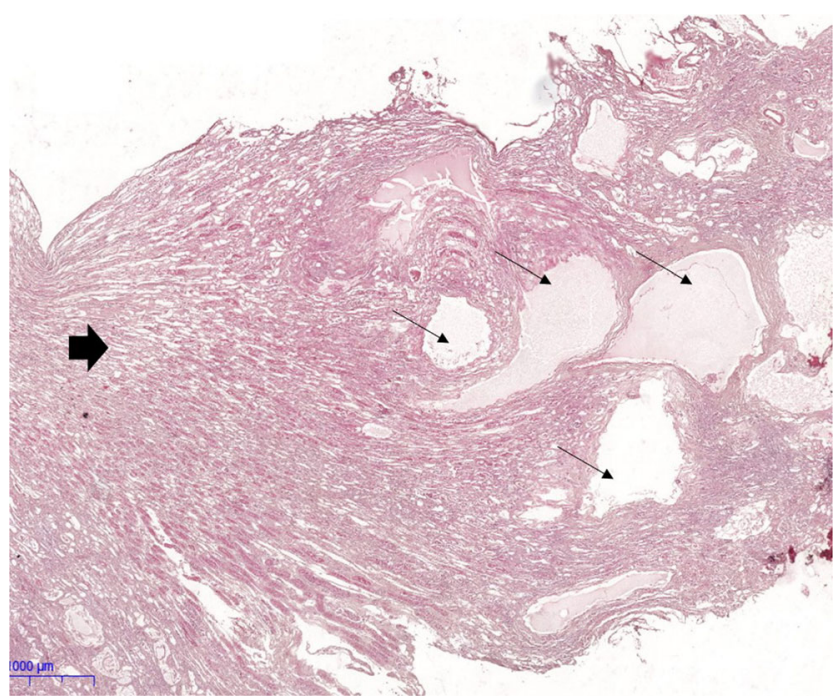

Fig. 3 Presence of multiple homogenous fluid-filled cysts (arrows) in the renal parenchyma and loss of renal parenchyma structural details (solid arrow) $(2 \times)$. Haematoxylin and eosin. Barr $=1000 \mu \mathrm{m}$

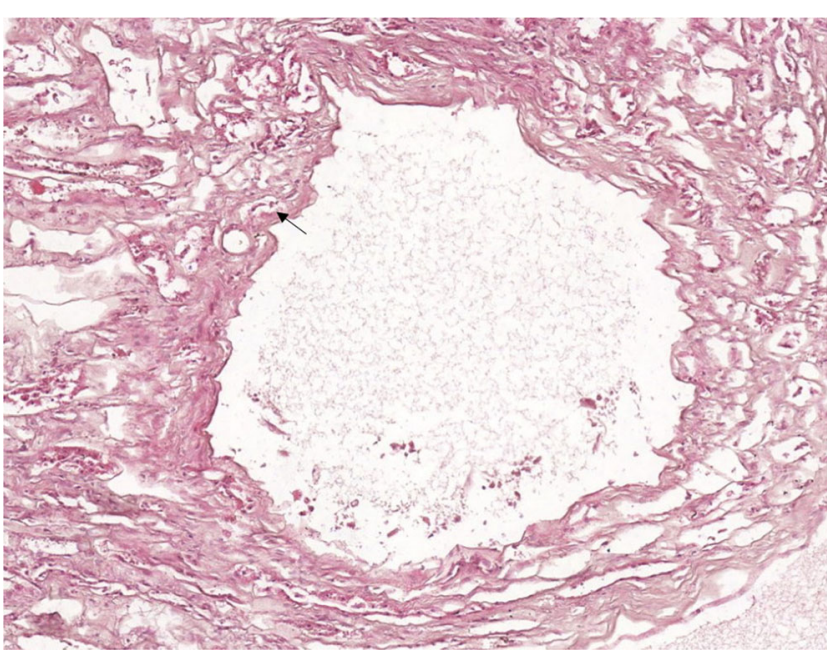

Fig. 4 The cyst was lined by a single layer of squamous epithelial cell (arrow) $(10 \times)$; haematoxylin and eosin. Barr $=100 \mu \mathrm{m}$

well as endothelial cell-mediated vasodilatation impairment (Adamczak et al. 2002). Increase in blood pressure is mediated by an increase in cardiac output. When the heart fails to pump blood at a sufficient rate to supply oxygen of all vital organs, it results in compensatory left ventricular hypertrophy (Cannon 2016), as seen in the gross lesion of this case report. The entire event will eventually lead to congestive heart failure (CHF). Renal damage also causes decreased production of erythropoietin that affects erythropoiesis in the bone marrow, causing anaemic condition in the cat (Chalhoub et al. 2011).

The diagnosis of polycystic kidney disease (PKD) is mainly through knowing breed susceptibility, the manifestation of clinical signs, complete blood count, serum biochemical profile, urinalysis, ultrasonography examination, SDMA test, urine protein creatinine ratio and radiography findings. For the present case, the clinical signs of polyuria and polydipsia, blood results of normochromic and macrocytic anaemia, hypoproteinaemia, elevated urea and creatinine levels narrowed down the tentative diagnosis into kidney disease.

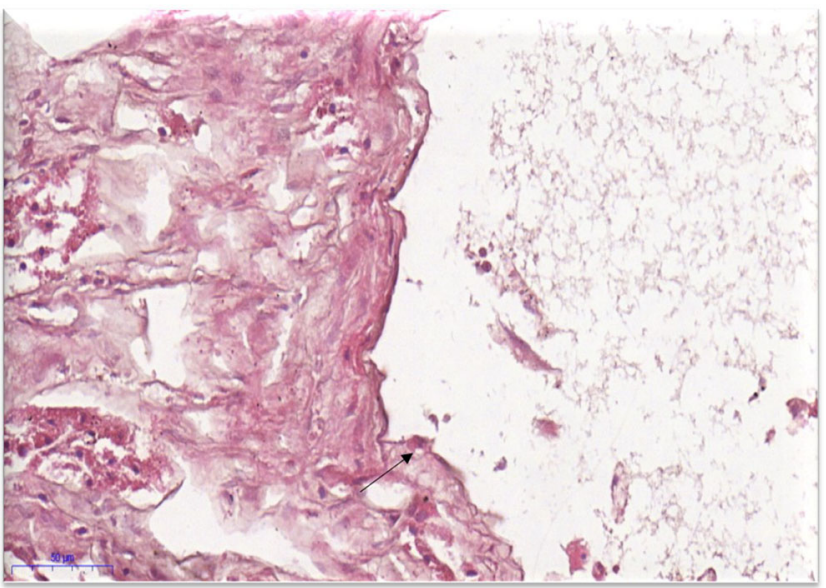

Fig. 5 Some squamous epithelial cells (arrow) of the cyst are necrotised due to pressure necrosis $(20 \times)$. Haematoxylin and eosin. Barr $=50 \mu \mathrm{m}$ 


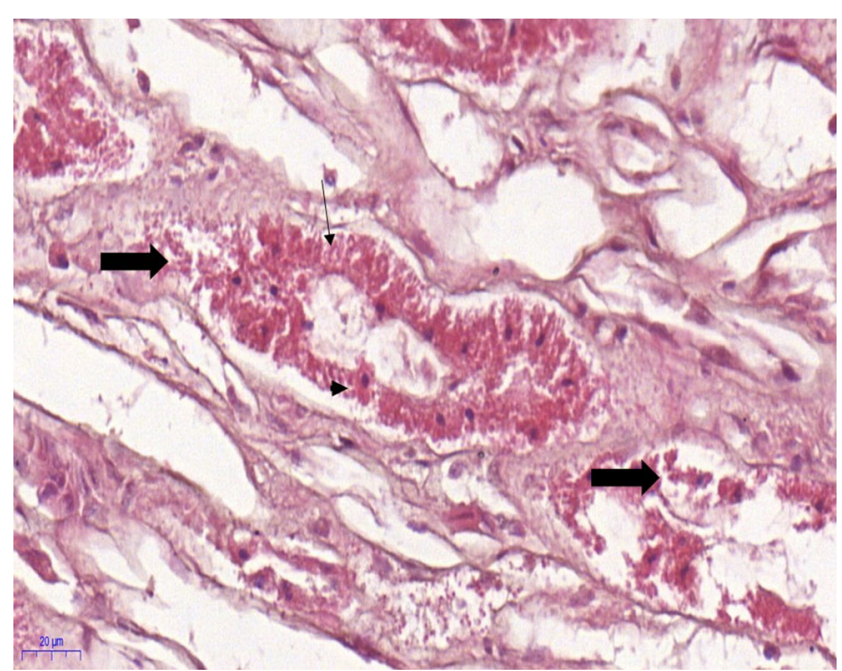

Fig. 6 The renal tubular epithelial cells underwent pyknosis (arrowhead) and karyolysis (arrow) while some tubular cells had undergone hydropic degeneration (solid arrow) $(40 \times)$. Haematoxylin and eosin. Barr $=20 \mu \mathrm{m}$

Polycystic kidney disease-induced chronic kidney disease was definitively diagnosed based on the post-mortem findings of typical multiple polycystic lesions found on both sides of the renal parenchymal as well as the histopathological lesions of homogenous fluid-filled cyst lined by a single layer of epithelial cell. Pressure necrosis exerted by the space-occupying lesions had caused distortion of normal renal parenchymal and loss of renal architecture and thus impair the function of the kidney and lead to chronic kidney disease. PKD can also be diagnosed through genetic testing for PKD1 gene mutation (Lee et al. 2010). Treatment for cats diagnosed with PKD includes ultrasound-guided drainage of the renal cyst, symptomatic and supportive treatments and dietary management. Several drug therapies used to control the disease had been done for human PKD case but not in the cat. These drugs are rapamycin complex inhibitors (sirolimus and everolimus), somatostatin analogues and bosutinib, which might help to slow the cyst growth (Rangan and Nankivell 2014).

Any concurrent infection worsens the condition of PKD and CKD in the animal. Feline parvovirus is known to replicate in actively dividing cells such as lymphoid tissue and intestinal villi. The virus infection results in losses of epithelial cells and shortened intestinal villi that cause impaired nutrition absorption (Sykes 2014). It results in diarrhoea that often contains blood and mucus due to loss of osmotic regulation. Besides, parvovirus infection also causes immunosuppression due to its effect on the lymphoid tissue, which leads to lymphopenia due to lymphocytolysis (Parrish 1995; Truyen et al. 2009). The immunocompromised cat may develop bacteria and septicaemia further exacerbates the diseased condition in the animal. As observed in the present case, the cat was tentatively diagnosed with feline parvovirus infection due to episodes of watery diarrhoea and further confirmed with parvovirus rapid test kit. The broad spectrum and extended antibiotic treatments were immediately administered against the secondary bacterial infection due to parvovirus infection and immunosuppressed state of the patient resulting from chronic disease.

\section{Conclusion}

Polycystic kidney disease (PKD) is an inherited disease which eventually causes the development of chronic kidney disease (CKD). Concurrent parvoviral and bacterial infections complicate the disease and cause various organ failures and death to the animal.

Acknowledgements The authors would like to acknowledge the staff of Universiti Malaysia Kelantan Veterinary Clinic, Histopathology and Bacteriology Laboratory, Faculty of Veterinary Medicine, Universiti Malaysia Kelantan, for their technical assistance while handling this case.

\section{Compliance with ethical standards}

Conflict of interest The authors declare that they have no conflict of interest.

Ethical approval All applicable international, national and/or institutional guidelines were followed.

\section{References}

Abdallah AA, El-Deen NAN, Neamat-Allah AN, Abd El-Aziz HI (2020a) Evaluation of the hematoprotective and hepato- renal protective effects of Thymus vulgaris aqueous extract on thermally oxidized oil-induced hematotoxicity and hepato-renal toxicity. Comp Clin Pathol 29(2):451-461

Abdallah AA, Nasr El-Deen NA, Abd El-Aziz HI, Neamat-Allah AN (2020b) Effect of the aqueous root extract of Curcuma longa L. (turmeric) against thermally oxidized oil-induced hematological, biochemical and histopathological alterations. Comp Clin Pathol 2020:1-9

Adamczak M, Zeier M, Dikow R, Ritz E (2002) Kidney and hypertension. Kidney Int 61:62-67

Barrs VR (2019) Feline Panleukopenia. Vet Clin N Am Small Anim Pract 49(4):651-670

Barrs VR, Gunew M, Foster SF, Beatty JA, Malik R (2001) Prevalence of autosomal dominant polycystic kidney disease in Persian cats and related-breeds in Sydney and Brisbane. Aust Vet J 79(4):257-259

Brown CA, Elliott J, Schmiedt CW, Brown SA (2016) Chronic kidney disease in aged cats: clinical features , morphology , and proposed pathogeneses. Vet Pathol 53(2):309-326

Cannon M (2016) Diagnosis and investigation of chronic kidney disease in cats. In Pract 38:2-9

Chalhoub S, Langston CE, Eatroff A (2011) Anemia of renal disease: what it is, what to do and what's new. J Feline Med Surg 13(9):629 640

Hartmann K (2017) Feline panleukopenia update on prevention. Thai J Vet Med 47:101-104

Lee YJ, Chen HY, Hsu WL, Ou CM, Wong ML (2010) Diagnosis of feline polycystic kidney disease by a combination of 
ultrasonographic examination and PKD1 gene analysis. Vet Rec 167(16):614-618

Lyons LA, Biller DS, Erdman CA, Lipinski MJ, Young AE, Roe BA, Qin B, Grahn RA (2004) Feline polycystic kidney disease mutation identified in PKD1. J Am Soc Nephrol 15(10):2548-2555

Neamat-Allah AN, Abd El Haki Y, Mahmoud EA (2020) Alleviating effects of $\beta$-glucan in Oreochromis niloticus on growth performance, immune reactions, antioxidant, transcriptomics disorders and resistance to Aeromonas sobria caused by atrazine. Aquac Res 51(5):1801-1812

Parrish CR (1995) 3 pathogenesis of feline panleukopenia virus and canine parvovirus. Baillieres Clin Haematol 8(1):57-71

Phoon MHE, Khor KH, Lau SF, Saw MH (2015) Polycystic kidney disease in a Persian cat. J Vet Malaysia 27(1):20-23

Rangan GK, Nankivell BJ (2014) Management of autosomal dominant polycystic kidney disease. Med Today 15(6):16-28
Sykes JE (2014) Feline panleukopenia virus infection and other viral enteritides. In. Canine and feline infectious diseases, Elsevier, pp 187-194. https://doi.org/10.1016/B978-1-4377-0795-3.00019-3.

Truyen U, Addie D, Belák S, Boucraut-Baralon C, Egberink H, Frymus T, Gruffydd-Jones T, Hartmann K, Hosie MJ, Lloret A, Lutz H (2009) Feline panleukopenia. ABCD guidelines on prevention and management. J Feline Med Surg 11(7):538-546

Volta A, Manfredi S, Gnudi G, Gelati A, Bertoni G (2010) Polycystic kidney disease in a Chartreux cat. J Feline Med Surg 12(2):138-140

Wills SJ, Barrett EL, Barr FJ, Bradley KJ, Helps CR, Cannon MJ, Gruffydd-Jones TJ (2009) Evaluation of the repeatability of ultrasound scanning for detection of feline polycystic kidney disease. J Feline Med Surg 11(12):993-996

Publisher's note Springer Nature remains neutral with regard to jurisdictional claims in published maps and institutional affiliations. 